\title{
Extrapericardial bronchogenic cyst associated with atrial fibrillation
}

\begin{abstract}
Bronchogenic cysts are rare and usually asymptomatic. There are very few reports of bronchogenic cysts producingatrial fibrillation in the literature. We present a rare case of atrial fibrillation as a complication of a mediastinal bronchogenic cyst. To our knowledge, there have been only 13 cases of atrial fibrillation caused by a bronchogenic cyst published in the literature. As demonstrated by prior studies, surgical resection has been a successful treatment of the atrial fibrillation in this population. Our case report presents additional evidence to support the effectiveness of surgical resection in the treatment of bronchogenic cyst induced atrial fibrillation.
\end{abstract}

Volume II Issue 6 - 202I

\author{
Andrei I Gritsiuta, Alexander Bracken, Jorge \\ Lara-Gutierrez \\ Department of Surgical Services, University of Pittsburgh \\ Medical Center, USA
}

\begin{abstract}
Correspondence: Andrei I Gritsiuta, Department of Surgical Services, University of Pittsburgh Medical Center 3600 Forbes Avenue, USA, Email gritsiutaai@upmc.edu
\end{abstract}

Received: November 0I, 202I | Published: November I5, 202

\section{Introduction}

A Bronchogenic cyst is a rare congenital intrathoracic lesion that accounts for approximately $5 \%-20 \%$ of primary mediastinal masses. ${ }^{1-3}$ Themajority of cysts are asymptomatic or have a nonspecific clinical presentationdue tothe compression of surrounding structures. ${ }^{4}$ Cardiovascular complications have rarely been reported and are usually due to an intrapericardial location of the cystic mass. ${ }^{3}$ In this article, we present a patient with an extrapericardial bronchogenic cyst that manifested with new-onset paroxysmal atrial fibrillation with rapid ventricular response (RVR). The arrhythmia was caused by direct compression on the left atrium and pulmonary vein and was managed by performing a surgical cyst excision. Patient has remained asymptomatic for 15 months postoperatively.

\section{Case report}

A 43-year-old African American female presented with progressively worsening chronic nonproductive cough, shortness of breath and palpitation without chest pain, lightheadedness or syncopal episodes. She was recently diagnosed with new-onset paroxysmal atrial fibrillation (AF) three month prior to presentation and was prescribed Eliquis for thrombosis prophylaxis. On presentation, an ECG revealed atrial fibrillation with RVR with a rate of $150 \mathrm{bpm}$ and prolonged QT interval. A Chest CT scan demonstrated a large $43 \times 44 \times 50$ mmposterior mediastinal cystic lesion in the right infrahilar region causing a mass effect on the bronchus intermedius as well as the left atrium. The appearance of the mass on CT was consistent with abronchogenic cyst (Figure 1). The patient's new-onset AF was attributed to the compression of left atrium and pulmonary vein. Patient was prescribed flecanide and subsequently pharmacologically converted to sinus rhythm.

After rhythm stabilization, stress test and cardiology clearance, patient was taken to the operating room for abronchogenic cyst excision. During bronchoscopy, no endobronchial lesions were observed; however, the right bronchus intermedius was almost completely collapsed due to external compression. A VATS procedure was then attempted. Findings demonstrated alarge firm cystic structure in the posterior mediastinum that was compressing and intimately adherent to the subcarinal region, right lower lobe and extended to the pulmonary vein with extensive pericystic inflammation and fibrosis. Due to dense adhesions and inability to safely isolate the cystic lesion, the procedure was converted to a thoracotomy. Dissection proceeded and the cyst wall was freed from surrounding structures employing extreme caution to prevent damage of adherent anatomy and avoiding cautery adjacent to the airway. The entire cyst was excised and sent to pathology.
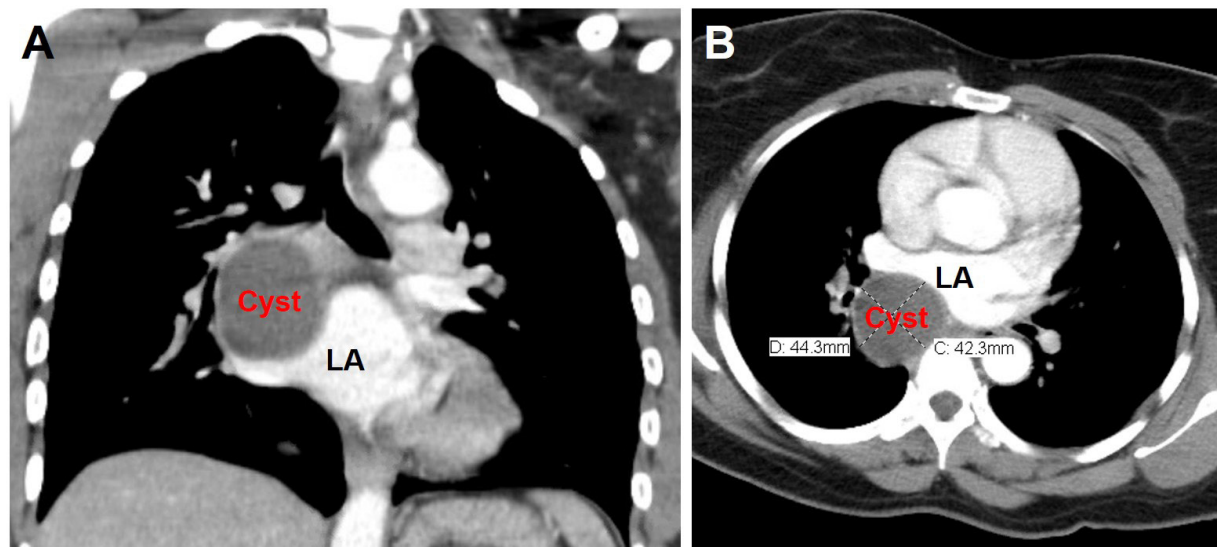

Figure I Chest CT images showing large homogeneous, low-density, unilocular, well circumscribed mass $43 \mathrm{~mm} \times 44 \mathrm{~mm} \times 50 \mathrm{~mm}$ (I 7 to $20 \mathrm{Hounsfield} \mathrm{units),} \mathrm{with}$ direct extrinsic compression of the left atrium. (A) coronal and (B) axial views. 
Pathologic interpretation was confounded by cystic alterations noted by inflammation, hemorrhage and fibrosis. Secondary to these features, and lack of cartilage within the cystic wall, the cyst was initially thought to represent a thymic cyst. Additional features of the specimen demonstrated largely denuded ciliated columnar epithelial lining of the cyst. After further review, final pathology was determined to be consistent with inflamed and altered bronchogenic cyst.

Postoperatively patient begin ambulating on postoperative day 1 and chest tube was removed. The patient was discharged home on postoperative day 2 in normal sinus rhythm with a prescription of flecanide. Follow-up ECGs performed at 1, 5 and 12months have demonstrated normal sinus rhythm and patient continued to be asymptomatic at 15 months.

\section{Discussion}

A Bronchogenic cyst is a rare congenital benign neoplasm of primitive foregut origin that embryologically separates from a normal tracheobronchial tree during the third week of gestation. Bronchogenic cysts most commonly occur in the mediastinum adjacent to the major airways. ${ }^{3-6}$ The classic histological findings associated with bronchogenic cysts are ciliated columnar epithelial cells that comprise the inner lining along with thick walls that typically contain cartilage and bronchial mucous glands, however these features are not essential for the diagnosis. ${ }^{1,7}$

Most cysts in adults are asymptomatic and incidentally identified on radiography; nevertheless, they can present when complications occur. ${ }^{4,8}$ The clinical presentation depends on the location of the cyst and ranges from nonspecific symptoms such as cough, chest pain and/or dyspnea, to severe life-threatening conditions secondary to infection, hemorrhage, or cysticrupture. ${ }^{5}$ Enlarging lesions become symptomatic due to direct compression of adjacent anatomical structures. Cardiovascular complications are extremely rare and include various types of arrhythmias, sudden cardiac death, cardiac tamponade, as well as SVC syndrome. ${ }^{1,3,7,10}$ Stretching of the pulmonary vein is known to triggerirregular neurotransmission of cardiomyocytes. ${ }^{5}$ The insidious onset of atrial fibrillation in a young individual without underlying cardiac pathology should raise suspicion of a bronchogenic cyst. Thestandard diagnostic workup of a bronchogenic cyst should include plain radiography, echocardiography, and a CT scan of the chest. In the present case, the new onset of atrial fibrillation was due to direct compression of left atrium and pulmonary vein. Only 13 similar cases of atrial fibrillation secondary to a bronchogenic cystic have been described in the English literature(Table 1) ${ }^{1-13}$ The gold standard for management of cardiac complications associated with cystic lesions is surgical resection. The most common approach for resection is thoracotomy which has been demonstrated by the $77 \%$ of the cases described in the literature. This approach is performed in the majority of cases due to extensive fibrosis and inflammation. ${ }^{2,4-11,13}$ VATS or a robotic-assisted approach is an alternative surgical option for decompression or excision of a bronchogenic cyst. ${ }^{3,12}$ Complete resection of the cystic wall is crucial because there is increased likelihood of cyst recurrence and malignant transformation following incomplete excision. ${ }^{1,12}$ hence, complete cyst removal is recommended even if the patient is asymptomatic..$^{6-8,10}$ In poor surgical candidates, endobronchial ultrasound-guided transbronchial needle aspiration (EBUS-TBNA), transthoracic CTguided aspiration or mediastinoscopy may provide an alternate treatment strategy. ${ }^{1,8,13}$

Table I Extrapericardial bronchogenic cysts associated with new-onset atrial fibrillation

\begin{tabular}{|c|c|c|c|c|c|}
\hline Author & Year & Age & Sex & Treatment & Follow-up \\
\hline Volpi et al. ${ }^{4}$ & 1988 & 36 & M & Thoracotomy & - \\
\hline Johnston et al. ${ }^{8}$ & 1992 & 44 & $\mathrm{~F}$ & CT-guided aspiration and thoracotomy & 16 months \\
\hline Mather et al. ${ }^{9}$ & 1995 & 50 & $\mathrm{~F}$ & Thoracotomy & - \\
\hline Sick et al." & 2000 & 61 & M & Thoracotomy & - \\
\hline Parambil et al. ${ }^{5}$ & 2006 & 38 & M & Thoracotomy & 12 months \\
\hline Marshall et al. ${ }^{7}$ & 2007 & 32 & M & Thoracotomy & - \\
\hline Fujino et al. ${ }^{6}$ & 2010 & 62 & M & Thoracotomy & 36 months \\
\hline Narula et al..$^{13}$ & 2013 & 53 & M & EBUS-TBNA and thoracotomy & - \\
\hline Alraiyes et al.' & 2015 & 67 & M & EBUS-TBNA & 3 months \\
\hline Smail et al. ${ }^{2}$ & 2015 & 66 & M & Mediastinoscopy and thoracotomy with deroofing & 24 months \\
\hline Han et al. ${ }^{10}$ & 2018 & 49 & $\mathrm{~F}$ & Thoracotomy & 60 months \\
\hline Liu et al. ${ }^{3}$ & 2018 & 29 & $\mathrm{~F}$ & Robotic-assisted VATS & - \\
\hline Nakamura et al. ${ }^{12}$ & 2019 & 83 & $\mathrm{~F}$ & Prone-position VATS with palliative fenestration & 18 months \\
\hline
\end{tabular}

\section{Data availability}

The data used to support the findings of this study are included within the article.

\section{Conflicts of interest}

All authors have no financial disclosures or conflicts of interest to disclose.

\section{Funding statement}

The research received no specific grants from any funding agency in the public, commercial, or non-for-profit sectors.

\section{Informed consent}

Written/Verbal informed consent was obtained from all individual participants included in the study. 


\section{References}

1. AH Alraiyes, K Shaheen, J Reynolds, et al. Recurrent Bronchogenic Cyst After Surgical Resection. The Ochsner journal. 2015; 15(2):176-179.

2. H Smail, Baste, Jean Marc, et al. Mediastinal Bronchogenic Cyst With Acute Cardiac Dysfunction: Two-Stage Surgical Approach. The Annals of thoracic surgery. 2015;100(4):79-e80.

3. Q Liu, Vainrib AF, Aizer A, et al. Multimodality Imaging of a Rare Case of Bronchogenic Cyst Presenting as New-Onset Atrial Fibrillation in a Young Woman. CASE (Philadelphia, Pa.). 2018;2(6):254-257.

4. A. Volpi, Cavalli A, Maggioni AP, et al. Left atrial compression by a mediastinal bronchogenic cyst presenting with paroxysmal atrial fibrillation. Thorax. 1998;43(3):216-217.

5. Parambil JG, Krowka MJ, Ryu JH, et al. Bronchogenic Cyst Causing Atrial Fibrillation by Impinging the Right Inferior Pulmonary Vein. The American journal of the medical sciences. 2006;331(6):336-338.

6. Fujino S, Hwang E, Sekido N, et al. Paroxysmal Atrial Fibrillation due to Bronchogenic Cyst, Internal medicine (Tokyo, 1992). 2010;49(19):21072111
7. Marshall G, C Cheah, Lenzo N, et al. Bronchogenic cyst with multiple complications, Biomedical imaging and intervention journal. 2007;3( 4)-e42.

8. Johnston SR, Adam A, Allison DJ, et al. Recurrent respiratory obstruction from a mediastinal bronchogenic cyst. Thorax. 1992; 47(8):660-662.

9. Mather E, Hogg J, Miller A. Covert bronchogenic cyst as a cause of lifethreatening cardiopulmonary impairment. Postgraduate medical journal. 1995;71(836): 369-371.

10. Han SJ, Cho HJ, Kang M, et al. A Life-Threatening Bronchogenic Cyst. Korean J Thorac Cardiovasc Surg. 2018;51(1): 69-71.

11. Sick P, Baldauf G, Schuler G. Mediastinal space-occupying lesion as a rare cause of atrial arrhythmia. Zeitschrift für Kardiologie. 2000;89(9):781787.

12. T Nakamura, R Fujikawa, Y Arai, et al. Palliative fenestration for a symptomatic subcarinal bronchogenic cyst by the prone position approach. Journal of surgical case reports. 2019;(12) rjz372.

13. Narula T, Sethi S, Raja S, et al. Bronchoscopic Therapy for Atrial Fibrillation - Case of a Large Bronchogenic Cyst. Chest. 2015;144(4): 201A-201B. 